\title{
Impaired mechanism of visual focal attention in posterior cortical atrophy
}

Silvia Primativo ${ }^{1}$, Sebastian Crutch ${ }^{2}$, Ivanna Pavisic ${ }^{2}$, Keir Yong ${ }^{2}$, Alessia Rossetti ${ }^{3}$, Roberta Daini ${ }^{3,4}$

${ }^{1}$ LUMSA University, Department of Human Science, Rome, Italy.

${ }^{2}$ University College of London, Queen Square Institute of Neurology, Dementia Research Centre, London, UK.

${ }^{3}$ Università degli studi di Milano-Bicocca, Milan, Italy.

${ }^{4}$ NeuroMI - Milan Center for Neuroscience and COMiB - Optics and Optometry Research Center, Milan, Italy.

Address for correspondence:

Silvia Primativo

Università LUMSA, Dipartimento di Scienze Umane

Piazza delle Vaschette 101, 00193, Roma

Telephone: +390668422235

Email: s.primativo@lumsa.it 


\begin{abstract}
Objective: Simultanagnosia, a deficit in holistic visual perception, is among the most prominent features of posterior cortical atrophy (PCA). Deficits in visuo-perceptual and attentional mechanisms could contribute to simultanagnosia. In the present study, we explored the impaired visual perception of global configuration with two main hypotheses: 1) it is due to a deficit in processing low-spatial frequency stimuli; 2) it arises from deficits in adjusting attentional focus.

Method: The visuo-perceptual mechanism was explored by asking participants (5 PCA patients and 20 age- and education-matched healthy controls) to report the local and global elements of incongruent hierarchical letters. Stimuli were unbiased (black letters/white background) and parvocellular-biased (red letters/green background). A cued T-detection task, where the stimulus onset asynchrony and the cues' features varied, was used to explore focal attention.
\end{abstract}

Results: PCA patients systematically failed in reporting the global, but not the local element. The parvocellular-biased condition partially improved the performance in only one patient. In the Tdetection task, controls responded faster to targets cued by red dots and small cues as compared to no cues. Conversely, the cue's features did not affect patients' performance.

Conclusions: Results only partially support the hypothesis according to which simultanagnosia is driven by an impairment in processing low-spatial frequencies. Data indicate a deficit in the flexibility of focal attention that prevents PCA patients from adapting the attentional window to the stimulus features. Simultanagnosia in PCA can be conceptualized as a complex result of a deficit involving visuo-perceptual and exogenous attentional mechanisms.

Keywords: Posterior cortical atrophy, simultanagnosia, focal attention, visuo-perceptual processing.

Key points: Question: We explored the mechanisms underlying the difficulties of patients with Posterior Cortical Atrophy in seeing more than one thing at the time. Findings: We report the role of perceptual and attentional factors. Importance: Results contribute to the knowledge of the cognitive mechanisms involved in a neuropsychological condition. Next steps: We will further investigate the reciprocal influence of the cognitive mechanisms in leading to individual differences in patients with posterior cortical atrophy. 


\section{Introduction}

Posterior cortical atrophy (PCA) is a progressive neurodegenerative syndrome mainly characterized by progressive dysfunctions of visual perception and visuo-spatial attention in a profile of relatively preserved episodic memory, insight, and judgment (Benson, Davis, \& Snyder, 1988). In addition, individuals with PCA often manifest alexia, dysgraphia, acalculia, apraxia and some or all of the features of Balint's syndrome such as simultanagnosia, oculomotor apraxia, optic ataxia, and environmental agnosia (Mendez et al., 2002; Renner et al., 2004; Tang-Wai et al., 2004; Charles \& Hillis, 2005; McMonagle, Deering, Berliner, \& Kertesz, 2006; Lehmann et al., 2011). The most frequent underlying pathology is Alzheimer's disease (AD), with PCA patients showing a greater distribution of senile plaques and neurofibrillary tangles in posterior regions of the parietal cortex, the occipital cortex and temporo-occipital junction relative to more anterior cortical areas (TangWai et al., 2004). Neuroimaging and neuropsychology tests can discriminate between the typical amnestic AD and PCA (Aresi \& Giovagnoli, 2009).

Simultanagnosia is characterized by the impairment in seeing several things at once, in identifying multiple stimuli and in interpreting complex scenes (Duncan et al., 2003; Riddoch \& Humphreys, 2004; Mazza, 2017). Patients with simultanagnosia are able to identify single objects but fail if multiple items are displayed regardless of size (Kinsbourne \& Warrington, 1962). When using hierarchical stimuli (e.g., Navon letters) patients with simultanagnosia are able to correctly identify the local component but systematically fail in identifying the global one. This is the opposite to what is normally reported in healthy controls, who show the global precedence effect, i.e., the tendency to process the global gestalt earlier as compared to the single local elements that constitute it (Navon, 1969; Robertson, 1996; Navon, 2003). The cognitive mechanisms underlying simultanagnosia have been attributed to deficits in visuo-perceptual or attentional mechanisms. A 
combination of the two is also possible, although such an option has received limited systematic investigation.

The visuo-perceptual component of simultanagnosia in PCA has been explored by several studies. Thomas, Kveraga, Huberle, Karnath, \& Bar (2012) have conceptualized simultanagnosia as a deficit in processing low-spatial frequencies. Hughes et al., (1990) have shown that global precedence effect described above might depend on the ability to process low spatial frequencies (with coarse rather than fine grain resolution). Spatial frequency describes the periodic distributions of light and dark in an image. While high-spatial frequencies convey information about fine details and small features or objects, low-spatial frequencies correspond to features such as global shape and large features or objects. Low spatial-frequency stimuli preferentially bias activation of the magnocellular system that predominantly conveys information to the visual dorsal pathway, frequently damaged in PCA. In this vein, Thomas et al., (2012) have hypothesized that if the visual ventral pathway is enhanced through a visual manipulation of the stimuli, the global processing problem can be ameliorated. In order to test this hypothesis, they used parvocellular-biased hierarchical letters as compared to unbiased stimuli. Parvocellular-biased stimuli consisted of red-green chromatic stimuli: indeed, the cells of the parvocellular system, unlike those of the magnocellular system, are sensitive to chromatic contrast. They tested this hypothesis on two patients with mixed aetiology (a progressive condition in one case and an acute event in the other). Results showed that the visual manipulation improved the global processing, reducing simultanagnosia in the two patients. Authors concluded that simultanagnosia is caused by impaired processing of low-spatial frequencies that prevent the perception of the global configuration.

Other studies have taken into account the size, numerosity, and spatial distance between local elements. Huberle \& Karnath (2006) have systematically manipulated inter-element distances 
between individual objects. Authors reported, in their two patients with simultanagnosia, a relative benefit in terms of global processing in the condition of reduced inter-letter distance. One of the two patients showed a marked improvement when the inter-letter distance was $0.85^{\circ}(87.5 \%$ accuracy) as compared to an inter-letter distance of $2.55^{\circ}$ (10\% accuracy). The second patient showed only a marginal improvement (around 20\%) with smaller inter-letter distances. Dalrymple, Kingstone, \& Barton (2007) tested a patient with simultanagnosia using hierarchical Navon letters. The authors manipulated stimulus density and size and found that the global processing in their patient improved with smaller inter-letter spacing and smaller stimulus size. This result is in line with other results on healthy individuals, whose reaction times on the global processing decreased for smaller inter-letter spacing (Dalrymple et al., 2007). Similarly, Montoro, Luna, and Humphreys (2011) reported an improved global performance in identifying hierarchical stimuli when the local elements were dense rather than sparse.

The role of an attentional mechanism in explaining simultanagnosic behaviour has also been taken into account. Simultanagnosia has been explained as a deficit in the disengagement of attention from one object (Pavese et al., 2002). The authors demonstrated that, in a two-stimuli array, only the offset of one of two stimuli allows the identification of the second one in a simultanagnosic patient. The visual offset of the first object, indeed, triggers a disengagement of attention, allowing the shift of attention to the other object. This interpretation is, however, somehow difficult to reconcile with the evidence of improved performance with dense rather than sparse local elements in hierarchical stimuli reported above (Dalrymple et al., 2007; Montoro et al., 2011). On the other hand, Dalrymple et al., (2010) and Dalrymple et al., (2011) have interpreted the impaired exploration of complex scenes in their simultanagnosic patients as the consequence of a restricted spatial area of visual processing. Similarly, Stark, Grafman, and Fertig (1997) hypothesized a "restricted spotlight of attention" in a patient with generalized brain atrophy due to Alzheimer's 
disease and a marked deficit in visual global processing. The described patient had a better performance in recognizing smaller vs. larger objects, in reading smaller vs. larger font-size words and in processing the local element of hierarchical stimuli. Likewise, evidence of better processing of smaller than larger letters, numbers (Crutch et al., 2011) and words (Yong, Rajdev, Shakespeare, Leff, \& Crutch, 2015) in PCA have been reported and interpreted as relating to a 'reduced effective field of vision'.

Another attentional mechanism that could be involved in simultanagnosia, and that has not yet been directly tested, is focal attention. This is a component of visuo-spatial attention and refers to the ability to change the size of the attentional window according with the dimensions of the stimulus to process (Eriksen \& Yeh, 1985; Eriksen \& James, 1986; Castiello \& Umiltà, 1990; Maringelli \& Umiltà, 1998; Turatto et al., 2000; Chun, Golomb, \& Turk-Browne, 2011; Albonico, Malaspina, Bricolo, Martelli, \& Daini, 2016; Albonico, Malaspina, \& Daini, 2017). It is not possible to conclude from the Stark et al. (1997) results whether the focus of attention is "restricted" or rather impaired in being adjusted to the size of the stimulus to be processed. Some evidence showed that, in healthy individuals, focal attention reduces the crowding phenomenon, in which object identification is inhibited by the presence of surrounding visual clutter (Albonico et al., 2018). Such a result suggests that focal attention could favour the segregation of distinct objects before the stage of feature integration, and help a correct visual identification. A deficit of focal attention should involve a low performance either with very small and closed stimuli (e.g., reading) or with complex stimuli made by many separate parts (e.g., visual scene and Navon hierarchical stimuli) because in both cases the attentional window could not adapt to the different sizes. We verify this hypothesis by means of a focal attention task (adapted from Maringelli \& Umiltà, 1998). 
In the present paper we investigated the perceptual and attentional mechanisms involved in simultanagnosia in posterior cortical atrophy with two main hypotheses: 1) it is due to an impairment in processing low-spatial frequencies as proposed by Thomas et al. (2012); 2) an impaired focal attention mechanism hinders an appropriate holistic perception of the stimuli.

In Experiment 1, we tested the low-spatial frequency hypothesis of simultanagnosia by comparing the PCA patients and controls' performance with chromatic and achromatic hierarchical stimuli. In Experiment 2, we assessed the participants' ability to name single letters of different sizes in order to exclude a more basic letter identification deficit. In Experiment 3, the focal attention hypothesis has been tested with the prediction that cue features will impact on the performance of healthy controls (Albonico et al., 2016, 2017, 2018) but not on PCA patients. Finally, in Experiment 4, by using a shape identification task, we controlled for the possible influence of a perceptual deficit on the focal attention mechanism.

\section{Method}

\section{Participants}

Five patients who met current criteria for a diagnosis of PCA owing to probable AD (Mendez et al., 2002; Tang-Wai et al., 2004; Crutch et al., 2017) took part in the study. The diagnosis was made based on clinical and neuroimaging data. Volume loss was more marked posteriorly in all participants with available MRI scans, consistent with a diagnosis of PCA. Patients were recruited at the Dementia Research Centre, UCL, London. Demographic information and the results of the neuropsychological testing are summarized in Table 1 . The clinical diagnosis of PCA can be formalized only in the absence of significant primary ocular disease explaining the symptoms 
(Mendez et al., 2002; Tang-Wai et al., 2004; Crutch et al., 2017). Accordingly, visual acuity was assessed in all five PCA patients using the Symbol Acuity test from the CORVIST (James, Plant, \& Warrington, 2001, details are provided below). The Snellen equivalent results for each patient are provided in Table 1 and indicated that four out of five patients were able to identify the smallest stimuli (Snellen equivalent 6/9). Only Patient 3 received a cataract operation 5 years before testing and showed a mild weakening of visual acuity (Snellen equivalent 6/18). Moreover, Patient 5 had left homonymous hemianopia.

Twenty neurologically healthy controls, matched with PCA patients for age and education $(p=0.2$ and 0.3 , respectively) have also taken part in the experimental procedures. Seven females and 13 males composed the control group (mean age of healthy controls was 65.8 years, s.d. $=3.9$, mean education was 17.3 years (s.d.=3.2). MMSE scores were within normal limits for all controls: average $=29.5$, s.d.= 0.7 . Healthy controls did not report any history of neurological and/or ophthalmological diseases and their visual acuity was normal or corrected to normal.

Ethic approval for the data collection was obtained from the institutions were the project was carried out. All participant signed an informed consent form. 
Table 1. Demographic information and neuropsychological tests scores for the five PCA patients.

\begin{tabular}{|c|c|c|c|c|c|c|}
\hline & Max score & Pt 1 & Pt 2 & Pt 3 & Pt 4 & Pt 5 \\
\hline Age & & 77.4 & 64.8 & 74.0 & 61.5 & 73.0 \\
\hline Gender & & $\mathrm{M}$ & M & $\mathrm{F}$ & M & $\mathrm{F}$ \\
\hline Years of education & & 17 & 17 & 16 & 16 & 17 \\
\hline Age at onset* & & 70.0 & 60.0 & 68.0 & 57.0 & 73.0 \\
\hline \multicolumn{7}{|l|}{ Neuropsychological Assessment } \\
\hline \multicolumn{7}{|l|}{ General and memory functions } \\
\hline MMSE & 30 & 29 & 29 & $20^{\wedge}$ & $27^{\wedge}$ & 30 \\
\hline Short Recognition Memory Test for words & 25 & 24 & 24 & 20 & 20 & 25 \\
\hline Concrete Synonyms & 25 & 21 & 23 & 24 & 21 & 24 \\
\hline Naming (verbal) & 20 & 20 & 19 & 18 & 19 & 19 \\
\hline Adapted Graded difficulty calculation test (GDA) & 26 & 19 & 18 & $10^{\wedge}$ & 19 & 19 \\
\hline Spelling (Baxter) & 20 & 15 & 20 & 14 & 14 & 20 \\
\hline Digit span Forward (max) & 12 & 7 & 8 & 7 & 7 & 9 \\
\hline Digit span Backward (max) & 12 & 4 & 4 & 3 & 5 & 5 \\
\hline \multicolumn{7}{|l|}{ Early visual processing } \\
\hline Visual acuity (CORVIST): Snellen & $6 / 9$ & $6 / 9$ & $6 / 9$ & $6 / 18$ & $6 / 9$ & $6 / 9$ \\
\hline Figure-ground discrimination (VOSP) & 20 & $16^{\wedge}$ & $18^{\wedge}$ & $10^{\wedge}$ & 19 & $10^{\wedge}$ \\
\hline Shape discrimination - Efron Squares & 20 & $12^{\wedge}$ & 19 & $10^{\wedge}$ & $15^{\wedge}$ & 19 \\
\hline Hue discrimination - CORVIST & 4 & $2^{\wedge}$ & $2^{\wedge}$ & $0^{\wedge}$ & $2^{\wedge}$ & 4 \\
\hline \multicolumn{7}{|l|}{ Visuo-perceptual processing } \\
\hline Object decision (VOSP) & 20 & $5^{\wedge}$ & $6^{\wedge}$ & $5^{\wedge}$ & $7^{\wedge}$ & $17^{\wedge}$ \\
\hline Unusual views & 20 & $0^{\wedge}$ & $2^{\wedge}$ & $0^{\wedge}$ & $4^{\wedge}$ & $6^{\wedge}$ \\
\hline Usual views & 20 & $3^{\wedge}$ & $14^{\wedge}$ & $8^{\wedge}$ & $18^{\wedge}$ & $18^{\wedge}$ \\
\hline Fragmented letters (VOSP) & 20 & $0^{\wedge}$ & $14^{\wedge}$ & $0^{\wedge}$ & $8^{\wedge}$ & 18 \\
\hline CORVIST reading test & 16 & 16 & 16 & 15 & 16 & 16 \\
\hline \multicolumn{7}{|l|}{ Visuo-spatial processing } \\
\hline Number location (VOSP) & 10 & $3^{\wedge}$ & 7 & $0^{\wedge}$ & $3^{\wedge}$ & $1^{\wedge}$ \\
\hline Dot counting ( $\mathrm{n}$ correct) & 10 & $3^{\wedge}$ & $6^{\wedge}$ & $4^{\wedge}$ & $6^{\wedge}$ & $6^{\wedge}$ \\
\hline As cancellation: number of missed letters & 19 & $7^{\wedge}$ & 1 & $11^{\wedge}$ & 0 & 2 \\
\hline
\end{tabular}

*Age at onset was assessed by asking participants or their caregivers when they first experienced symptoms.

$\wedge$ Pathological scores (below the $5^{\text {th }}$ percentile).

\section{Neuropsychological evaluation:}


1. Mini-Mental State Examination (MMSE, Folstein, Folstein, \& McHugh, 1975). It is a screening test consisting of a series of brief tasks. It involves several different mental abilities, including memory, attention, and language. Score range: 0-30.

2. Short Recognition Memory Test for words (Warrington, 1996). This test contains 25 words that are jointly visually and verbally presented at the rate of 1 every 3 seconds. Participants have to express a judgment about each one (pleasant or unpleasant). The participant is then shown (joint verbal and visual presentation) pairs of words: the target and a distractor. The task is to identify the already seen and heard word. Score range: 12 (chance) - 25.

3. Concrete synonyms test (Orpwood, 1998). The test is constituted of 25 target words verbally presented. Patients are required to indicate which one, out of two alternative words, is more similar in meaning to the target word. Score range: 12 (chance) -25 .

4. Naming - verbal description (adapted from McKenna \& Warrington, 1980). The experimenter describes some objects and other items, and the participant is required to name the target. The test is discontinued after four consecutive errors. Score range: 0-20.

5. Adapted Graded difficulty calculation test (Jackson \& Warrington, 1986). Patients are asked to sum up two numbers. The time cut-off is set to $30 \mathrm{~s}$ for items $1-12$ and $10 \mathrm{~s}$ for items $13-26$. The first 12 items are administered irrespective of performance. From item 13 the test is discontinued after three consecutive errors.

6. Spelling. (Baxter \& Warrington, 1985). Patients are asked to spell out loud 20 items. Mean length: 5.8 letters ( $S D=1.5$; range =4-10); mean CELEX frequency: 185.4 (SD=374.5; range =1-1464). The test is discontinued after four consecutive errors. 
7. Digit Span forward. Sixteen strings of numbers (length=2-9, two strings for each length) are presented to the patient at the rate of $1 / \mathrm{s}$. The patient is asked to repeat the string of numbers in the same order. The scores are represented by the total number of strings correctly reported and the maximum number of digits in a string that the patient could correctly recall. The task was discontinued after scores of 0 on both trials of an item. Score range: 2-9.

8. Digit Span backward. The test is administered as for the digit span forward. The only differences are the following: the patient is asked to repeat the sequence backward and the length of the strings is 2-7 digits, for a maximum of 14 strings administered. The task was discontinued after scores of 0 on both trials of an item. Score range: $2-7$.

\section{Early visual processing}

9. Visual acuity (CORVIST -James, Plant, \& Warrington, 2001). The test measures visual acuity using three different shapes (triangles, squares, and circles). Six rows, one for each size, each constituted of six items, are used. The score is given by the lowest row on which the participant responds to all six items accurately. Score range: 6/60-6/9.

10. Figure-ground discrimination (VOSP) (Warrington \& James, 1991). Patients are required to detect the presence of an $\mathrm{X}$ on speckled squares. Twenty items are used, and the target is present half of the times. Score range: 10 (chance score) -20 .

11. Shape discrimination - Efron squares (Efron, 1969). Patients are required to discriminate between a square and an oblong, which are randomly presented 10 times each. Score range: 10 (chance score) -20 .

12. Hue discrimination (CORVIST) (James et al., 2001). Patients are required to discriminate, among nine squares of similar colours, which of them is of a different hue. Score range: $0-4$. 
Visuo-perceptual processing

13. Object decision (VOSP) (Warrington \& James, 1991). Patients are required to identify, among four silhouettes, which one represent a real object (rather than a made-up one). The task was discontinued if the participant got a score of 3 or less in the first 10 items. Score range: 5 (chance score) -20 .

14. Usual/Unusual views (Warrington \& James, 1988). Patients are required to identify 20 pictures of objects taken from unusual perspective. Pictures taken from a usual perspective are represented for the objects that could not be identified from the unusual perspective. Score range: 0-20.

15. Fragmented letters (VOSP) (Warrington \& James, 1991). Patients are asked to identify twenty capital fragmented letters, presented one at the time. The task was discontinued if the participant got a score of 2 or less in the first 10 items. Score range: $0-20$.

16. Reading (CORVIST) (James et al., 2001). The test includes 16 words the patients are asked to read aloud. One point is given for each correctly read word. Score range: 0-16.

\section{Visuo-spatial processing}

17. Number location (VOSP) (Warrington \& James, 1991). Patients are required to look at two squares, exactly the same size. The top square contains numbers, all in different places. The bottom square just has a single dot in it. Patients are then asked to look at where the dot is in the bottom square, and indicate which number is in the same place in the top square. Ten trials are administered. The task was discontinued if the participant got a score of zero or less in the first five items. Score range: 0-10. 
18. Dot counting (VOSP) (Warrington \& James, 1991). Patients are required to indicate how many dots (range: 5-9) are represented on each of 10 pages. Reaction time is recorded for correct items. The task was discontinued if the participant got a score of zero or less in the first 5 items. Score range (accuracy): 0-10.

19. "A" cancellation (Willison \& Warrington, 1992). Participants are presented with an A4 sheet where 19 As are embedded among other $(\mathrm{N}=69)$ distractor letters. Participants' task is to mark all As. Maximum time allowed: $90 \mathrm{~s}$. The number of missed letters is reported.

As it can be observed in Table 1, the neuropsychological evaluation suggested that despite some mild individual differences, patients reported a relatively preserved performance in the tests assessing short-term episodic memory and other cognitive functions regarding language, calculation, spelling and working memory. The results of the early-visual processing tests indicated varying degree of impairment; patients 1 and 3 were impaired in all three tests, patients 2 and 4 were impaired in two tests and relatively preserved in one test and, finally, patient 5 was relatively less impaired and scored within normal levels in two out of three tests. The tests assessing the visuoperceptual processing indicated more homogeneous profiles; all patients were impaired in four out of five tests, with the exception of patient 5 that that was impaired in three out of five tests. All patients performed well in the CORVIST reading test. Finally, in terms of visuo-spatial processing, patients 1 and 3 were impaired in three out of three tests; patients 4 and 5 were impaired in two tests, while patient 2 was impaired in one test and scored within the normal ranges for the other two tests.

Overall, the neuropsychological examination revealed a relatively mild severity group of patients with reasonably intact primary visual functions and with visuo-perceptual and visuo-spatial deficits, core features of the PCA condition. The individual differences that can be observed among patients 
are part of the phenotypical continuum frequently reported in this population (Crutch et al., 2013; Firth et al., 2019).

\section{Data Analysis}

The experimental tests described below yield data of different nature that have been analysed accordingly. Experiments 1, 2 and 4 gave rise to accuracy data. In order to verify whether patients performed significantly worse than controls in perceptual tasks, and since we expected moderate to large variability in patients data, we planned to run appropriate Crawford analysis, suitable for this typology of neuropsychological data. In particular, we planned to apply a single-case approach adopting the case-control design following the procedure described in Crawford et al. (2010) for ttests. This procedure allows the estimation of the abnormality of a patient score in a task compared to a control sample modest in size, the significant level of such abnormality and the effect size (zcc). In Experiment 1, we also computed Yates corrected chi-squared tests for assessing, within each patient the impact of the experimental manipulation applied to the stimuli (parvocellular bias and letter fragmentation). Experiment 3 produced reaction times data. In order to evaluate if elderly controls data confirm the results of focal attention cue effects obtained with younger participants (Albonico et al., 2016), healthy controls data have been analysed via a two-way repeated-measures analyses of variance (ANOVA), with condition as independent variable and response time as dependent variable. Significant differences have been further explored by Bonferroni post-hoc multiple comparisons and the corrected $p$-values are reported. The effect size have been estimated by computing the Eta Squared values $(n 2)$. In order to verify whether patients performed significantly worse than controls, and since we expected large individual variability among PCA patients, RTs data of each individual PCA patient have been compared to the healthy controls' 
performance. To this aim, as for experiments 1, 2 and 4, the procedure described in Crawford et al. (2010) has been applied. All the statistical comparisons are driven by a-priori hypothesis therefore the significance level of $p$ values was set to 0.05 .

\section{Experimental tests}

\section{Apparatus}

Participants were seated in front of a 15 -inch computer monitor with a resolution of $1920 \times 1080$ pixels and a refresh rate of $60 \mathrm{~Hz}$. Viewing distance was constant at $57 \mathrm{~cm}$ by using a chin-rest. The experiment was controlled by the SR Research Experiment Builder software (SR Research Ltd., Canada). The same apparatus was used for all the experiments described below.

\section{Experiment 1 - Global and local processing of hierarchical Navon letters}

In Experiment 1, we tested for simultanagnosia in our participants using hierarchical letter stimuli. We also tested the low-spatial frequency hypothesis by comparing the participants' performance with chromatic (parvocellular-biased) and achromatic hierarchical stimuli. If a low-spatial-frequency deficit can account for simultanagnosia, an improvement in performance when using the parvocellular-biased manipulation is expected in patients, as suggested by Thomas et al. (2012). Moreover, since the patients' difficulty in identifying the global stimulus when it is made up of small letters could be ascribed to the global stimulus being fragmented, we can also expect a low accuracy rate in identifying local letters when these are fragmented.

\section{Stimuli and procedure}

In Experiment 1, we used hierarchical stimuli (Figure 1) that consisted of global patterns of three different letters $(E, H$, and $S)$ formed from smaller local patterns of the same letters. All possible 
combinations of incongruent stimuli (i.e., identity of the global and local shapes were different) were applied resulting in a total number of six different combinations. The overall stimulus' size was $15^{\circ} \times 15^{\circ}$ and the local letter's size was $1.2^{\circ} \times 1.2^{\circ}$. Inter-letter distance was $0.6^{\circ}$ (border to border). Half of the stimuli were parvocellular-biased (red target on a green background) and half were unbiased (black target on a white background). Half of the stimuli were constituted of fragmented local letters (see Figure 1).

Two separate experiments were run for global and local tasks. In the local task, the participant was asked to report the local or small letter, in the global task the participant was required to report the global or big letter. The order of presentation of the two experiments was counterbalanced across participants. Each experiment consisted of 40 trials (10 trials for each condition: unbiased unfragmented, unbiased fragmented, biased unfragmented, biased fragmented). Participants familiarized with the printed version of the stimuli and the experimental procedure was only started once the participant correctly responded to three consecutive items in both the global and the local tasks. Presentation time was unlimited and the stimulus disappeared when the participant responded. Controls were required to press the buttons on the keyboard. Patients' responses were instead inputted by the researcher due to visual disorientation. Accuracy was measured for each participant.

\section{***** Figure 1}

\section{Results and comments}

Controls performed very well in both the global and local task. As described above, the Crawford et al. (2010) procedure was applied to estimate, for each patient, the abnormality of the obtained score as compared to a group of healthy controls. 
As shown in Table 2, in the local task all but one patients (Patient 1) were accurate, showing no differences in performance as compared to controls. Conversely, in the global task, only one patient (Patient 5) was accurate, while the other four patients showed significantly poorer performance, with accuracy ranging from $3 \%$ to $78 \%$. 
Table 2. Controls' average and patients' individual results at the global and local Navon tasks (proportion of correct responses). For each patient the results of the statistical comparisons are reported ( $t$ test, $p$ value, effect size Z-CC score, respectively).

\begin{tabular}{|l|l|l|}
\hline & Overall local & Overall global \\
\hline Controls $(\mathrm{N}=20)$ & $0.98(\mathrm{sd}=0.03)$ & $0.99(\mathrm{sd}=0.02)$ \\
\hline & & \\
\hline Pt 1 & $0.85(\mathrm{t}=-4.3 ; \mathrm{p}=0.0002 ; \mathrm{Z}-\mathrm{CC}=-4.33)$ & $0.5(\mathrm{t}=-5.3 ; \mathrm{p}=0.00002 ; \mathrm{Z}-\mathrm{CC}=-5.44)$ \\
\hline Pt 2 & $1(\mathrm{t}=-0.6 ; \mathrm{p}=0.3 ; \mathrm{Z}-\mathrm{CC}=0.67)$ & $0.78(\mathrm{t}=-2.28 ; \mathrm{p}=0.02 ; \mathrm{Z}-\mathrm{CC}=-2.33)$ \\
\hline Pt 3 & $1(\mathrm{t}=-0.6 ; \mathrm{p}=0.3 ; \mathrm{Z}-\mathrm{CC}=0.67)$ & $0.03(\mathrm{t}=-10.41 ; \mathrm{p}<0.0001 ; \mathrm{Z}-\mathrm{CC}=-10.67)$ \\
\hline Pt 4 & $1(\mathrm{t}=-0.6 ; \mathrm{p}=0.3 ; \mathrm{Z}-\mathrm{CC}=0.67)$ & $0.63(\mathrm{t}=-3.9 ; \mathrm{p}=0.0005 ; \mathrm{Z}-\mathrm{CC}=-4.0)$ \\
\hline Pt 5 & $1(\mathrm{t}=-0.6 ; \mathrm{p}=0.3 ; \mathrm{Z}-\mathrm{CC}=0.67)$ & $1(\mathrm{t}=0.1 ; \mathrm{p}=0.9 ; \mathrm{Z}-\mathrm{CC}=0.1)$ \\
\hline
\end{tabular}

Within the global task, we explored the effect of the parvocellular bias manipulation. Given the large variability among patients, individual chi-squared tests between different experimental conditions were run (see Table 3 for individual results in the different experimental conditions). Out of the five patients, one was accurate ( $\mathrm{Pt} 5$ ) in terms of performance, so the difference between the parvocellular biased and unbiased condition, in this patient, was not explored. One out of four of the remaining patients (patient 4) showed a statistically significant improvement in terms of performance when the parvocellular bias manipulation was used. Two patients (patients 1 and 3 ) did not benefit from the perceptual manipulation, and one showed a significant worsening of the performance when the parvocellular bias manipulation was used (patient 2). Finally, two patients (patients 1 and 4) showed significantly poorer performance when fragmented letters were used. 
Table 3. Proportion of correct responses for the five PCA patients in the global condition of Experiment 1. The parvocellular bias effect is investigated through comparisons between unbiased and parvocellular bias unfragmented stimuli. The fragmentation effect is shown as the comparisons between fragmented and unfragmented unbiased stimuli. Yates corrected chi-squared test and $p$ values are reported.

\begin{tabular}{|c|c|c|c|c|c|c|}
\hline & \multicolumn{2}{|r|}{ Pt 1} & Pt 2 & Pt 3 & Pt 4 & Pt 5 \\
\hline \multicolumn{7}{|c|}{ Parvocellular bias effect } \\
\hline $\begin{array}{l}\text { Unfragmented } \\
\text { unbiased } \\
\text { (accuracy) } \\
\text { Unfragmented } \\
\text { parvocellular } \\
\text { biased } \\
\text { (accuracy) }\end{array}$ & 0 . & $\begin{array}{c}x^{2}=1.64 \\
p=0.2\end{array}$ & $\begin{array}{ll}0.8 & \\
& x^{2}=31.7 \\
& p<0.0001 \\
0.6 & \end{array}$ & $\begin{array}{l}0 \\
0\end{array}$ & $\begin{array}{ll}0.6 & \\
& X^{2}=52.8 \\
& p<0.0001 \\
0.9 & \end{array}$ & $\begin{array}{l}1 \\
1 \\
1\end{array}$ \\
\hline \multicolumn{7}{|c|}{ Fragmentation effect } \\
\hline $\begin{array}{c}\text { Unbiased } \\
\text { unfragmented } \\
\text { (accuracy) } \\
\text { Unbiased } \\
\text { fragmented } \\
\text { (accuracy) }\end{array}$ & 0 . & $\begin{array}{l}X^{2}=7.52 \\
p=0.006\end{array}$ & 0.8 & $\begin{array}{ll}0 & \\
& x^{2}=8.53 \\
& p=0.004 \\
0.1 & \end{array}$ & $\begin{array}{ll}0.6 & \\
& x^{2}=7.22 \\
& p=0.007 \\
0.4 & \end{array}$ & 1 \\
\hline
\end{tabular}

In summary, four out of five PCA patients showed simultanagnosia, with accurate performance in identifying the local element of hierarchical stimuli but failing in identifying the global letter. While the deficit has been attributed to a low-spatial-frequency deficit (Thomas et al., 2012), the present results suggest that the low-spatial frequency hypothesis per se does not fully explain the data. In our case, the parvocellular bias manipulation improved the performance only in one out of four patients, it worsened it in another one, and it did not affect the performance in the other two 
patients. The fragmentation of the local element further reduced accuracy in the global task in two PCA patients, suggesting that such a perceptual feature might also play a role in simultanagnosia.

\section{Experiment 2 - Single letter identification test}

PCA patients might show a deficit in identifying global stimuli but not the constituent local elements as a consequence of the stimulus size (i.e., "inverse-size effect", (Russell, Malhotra, \& Husain, 2004; Crutch et al., 2011; Yong et al., 2014)). The aim of Experiment 2 was to test the patients' ability to name single letters of different sizes. If the simultanagnosic difficulty in identifying the global stimulus is related to the larger size of the stimulus itself, it might be hypothesized that patients are impaired in identifying large but not small solid letters.

\section{Stimuli and procedure}

To test the stimulus size hypothesis, three solid letters were used as targets: $H, E, S$. Small and large stimuli's sizes corresponded to the global and local elements of the hierarchical stimuli (Experiment 1). Half of the stimuli were parvocellular biased, and half were unbiased. Forty trials were run. The order of presentation was randomized across participants. No fixation point was used in order to maximise the possibility to compare the performance to this test to that of the other tests. No time constraints were given. Presentation time was unlimited, and the stimulus disappeared when the participant responded. The participant's task was to identify the target letter. Controls were required to press the corresponding buttons on the keyboard. The researcher entered the patients' verbal responses due to visual disorientation. Accuracy was measured. 


\section{Results and comments}

All controls and patients correctly identified both global and local letters ( $\approx 100 \%$ correct). No further statistics were run. Since the dimensions of the small and large letters were matched to the hierarchical letters used in Experiment 1, we could exclude that stimulus size is, itself, the component causing the patients' deficit in identifying global letters in Experiment 1.

\section{Experiment 3 - Focal attention test.}

In Experiment 3, we considered the hypothesis that PCA patients are able to identify the local but not the global element of the Navon letters partly because of rigidity of the focal window of attention.

In order to verify this hypothesis, we measured the effect of different cues on target detection in PCA patients and controls. Following the procedure used in Albonico et al. (2016), we measured reaction times among three conditions and a baseline: 1) a red dot, as an optimal cue for the target position, but not the focal component, since it does not convey any information about the size of the target stimulus; 2 ) a small square, as the optimal cue for the focal component since it encloses the target stimulus without masking it, and conveys spatial information about the optimal field of integration necessary to detect the target stimulus; 3 ) a big square, as a non-optimal cue for the focal component, because it directs the focusing on a spatial area much larger than the target stimulus. A baseline condition where the target appearance was not pre-cued was also included. The cues' physical features matched the features of the stimuli used to assess simultanagnosia in Experiment 1. Indeed, the small cue had the same size of the local element of the hierarchical letters, and the big cue had the same size of the global element of the hierarchical letters. 
Stimulus onset asynchrony (SOA), i.e., the interval between the appearance of the cue and the target, was manipulated in order to explore both the exogenous and endogenous allocation of attention (Epstein et al., 1997). Indeed, according to Epstein et al. (1997), shorter SOAs evoke an exogenous and automatic orienting of attention, while longer SOAs elicit a more voluntary and endogenous orientation of attention. Moreover, Albonico et al $(2016 ; 2017)$ found that in young participants the deployment of focal attention in foveal vision is mainly exogenous, i.e., a stronger effect of the small square cue for shorter SOAs.

\section{Stimuli and procedure}

Target stimuli consisted of a capital letter T (font Sloan, colour black) of $1^{\circ} \times 1^{\circ}$ degree of visual angle oriented upright, while the cue could be represented by a red dot (diameter of $0.4^{\circ}$ ) or a small black square $\left(1.2^{\circ} \times 1.2^{\circ}\right.$, line thickness $\left.=0.1^{\circ}\right)$ or a big black square $\left(15^{\circ} \times 15^{\circ}\right.$, line thickness $\left.=0.1^{\circ}\right)$ or by no cue. Both the target stimulus and the cue were displayed on a grey background.

As compared to Albonico et al. (2016), only the foveal condition was run such that both the cue and the target were presented at the centre of the screen. The participants had to detect the presence of the target stimulus on the screen by pressing the space bar on the keyboard with the right index finger. The appearance of the target was preceded by one of the 3 possible cues or by the absence of any cue (baseline), and the SOA between the cue and the target could be either 100 or $500 \mathrm{~ms}$. The order of the cue type and SOA were randomised within the experiment.

Each trial started with a blank grey screen followed after 1000 ms by one of the three possible cues. As in the original Albonico et al. (2016)'s study, we chose not to display any fixation point to avoid giving an additional or confounding cue to participants. After the disappearance of the cue, the T target was presented and remained on the screen until the participant responded (or for a maximum of $2000 \mathrm{~ms}$ ), after which the target disappeared, and the next trial started. In this 
condition, both the target stimuli and cues were always presented at the centre of the screen. An example of the trial procedure is reported in Figure 2.

Every participant completed 48 experimental trials. Trials were randomised across participants and equally divided between the 100 and 500 ms SOA conditions, and between the four cue conditions.

\section{$* * * * *$ Figure $2 * * * * *$}

\section{Results and comments}

Reaction times (RTs, measured from the onset of the target until the response was pressed) were adopted as the dependent measure. Outliers (i.e., RTs exceeding the individual mean plus or minus 2.5 SDs) were excluded from the analyses and accounted for $2.4 \%$ in controls and $4.2 \%$ in patients. Healthy controls RTs were analysed via a two-way repeated-measures Analyses of Variance (ANOVA) and the effects of interest were those associated with the experimental manipulations that is, Cue (absence of cue, dot, small square and big square), SOA (100 and $500 \mathrm{~ms}$ ) and their mutual interaction. Results are reported in Table 4. A significant SOA by cue emerged in the control group $[F(3,921)=4.5, p=0.004 ; \eta 2=0.014]$. The interaction indicated that while in the $100 \mathrm{~ms}$ SOA no differences emerged among the different cue types, in the 500 ms SOA both the red dot and the small cue yielded significantly shorter RTs (355 and $351 \mathrm{~ms}$, respectively) as compared to the no cue condition (399 ms). The large cue condition (377 ms) was not significantly different from the other conditions. The two cues refer to two different attentional mechanisms and indicate that elderly healthy controls are sensitive to both orienting and focal attentional mechanism in a detection task. In the light of the statistical analysis, we calculated the focal attention index (i.e. the difference in RTs between no cue and small cue) and the orienting index (i.e. the difference in RTs between no cue and the dot cue) within the 500 SOA condition. As described above, we compared each patient's performance with the control group by following the procedure described in Crawford et al (2010). 
Results indicated that as compared to controls, only patient 1 showed significant focal and orienting cue benefits $(t(19)=30.55, p<0.0001$ and $t(19)=8.38, p<0.0001$, respectively), while patients $2,3,4$ and 5 did not benefit from such cues (all $p>0.1$ ).

The lack of cue effects in four out of five PCA patients suggests a difficulty in the modulation of the focal and orienting attentional mechanisms, which does not adequately adjust to the features of the stimulus to be processed or the different size of the cues.

Table 4. Results from Experiment 3 - focal attention test. Mean reaction times for controls and for each PCA patient with different cue types and SOA intervals. In brackets, standard deviations for controls are reported.

\begin{tabular}{|l|l|l|l|l|r|r|r|}
\hline & controls & pt 1 & pt 2 & pt 3 & pt 4 & \multicolumn{1}{l|}{ pt 5 } \\
\hline & \multicolumn{5}{|c|}{ SOA = 100 } \\
\hline no cue & $\mathbf{4 1 0 . 4 ( 7 1 . 3 )}$ & 577.7 & 619.3 & 577.6 & 443.5 & 532.3 \\
\hline big & $\mathbf{4 2 0 . 0 ( 6 8 . 7 )}$ & 580.8 & 687.8 & 575.5 & 523.8 & 471.0 \\
\hline dot & $\mathbf{4 3 0 . 6 ( 7 0 . 1 )}$ & 539.2 & 764.2 & 1323.7 & 452.5 & 468.3 \\
\hline small & $\mathbf{4 1 9 . 8 ( 7 1 . 8 )}$ & 599.3 & 745.8 & 1084.2 & 436.0 & 495.3 \\
\hline & & & \multicolumn{1}{|c|}{ SOA = 500 } & & \\
\hline no cue & $398.9(66.3)$ & 607.6 & 643.5 & 1042.5 & 527.3 & 493.8 \\
\hline big & $\mathbf{3 7 1 . 9 ( 8 0 . 4 )}$ & 488.7 & 741.0 & 1213.5 & 451.5 & 503.0 \\
\hline dot & $\mathbf{3 5 5 . 2 ( 7 1 . 2 )}$ & 522.7 & 808.3 & 1544.0 & 535.3 & 530.3 \\
\hline small & $\mathbf{3 4 9 . 8 ( 6 8 . 0 )}$ & 506.0 & 648.4 & 1020.5 & 492.5 & 702.8 \\
\hline
\end{tabular}




\section{Experiment 4 - Shape discrimination test}

In Experiment 4 a shape discrimination test has been administered to rule out the possibility that patients did not make appropriate use of the attentional cues used in Experiment 3 because of an impaired perception of the cues themselves.

\section{Stimuli and procedure}

Three shapes were used: a triangle, a square, and a circle. For each shape two sizes were used which corresponded to the cues used in Experiment 3: large $\left(15^{\circ} \times 15^{\circ}\right.$, line thickness $\left.=0.1^{\circ}\right)$ and small $\left(1.2^{\circ} \times 1.2^{\circ}\right.$, line thickness $\left.=0.1^{\circ}\right)$. Thirty-six items were administered, 12 of each shape; half were large and half were small in size. The order of presentation was randomized. The shapes were black on a grey background and were presented in the centre of the screen. No fixation point was used in order to maximise the possibility of comparison with Experiment 3. No time constraints were given. The participant's task was to say if the target was a square, a triangle, or a circle. Verbal responses were inputted by the researcher, and only accuracy (not reaction times) was taken into account for the analysis.

\section{Results and comments}

Controls did not make any error in this test. Similarly, all patients were accurate (patients 1, 2 and 4) or made one single error (patients 3 and 5), so no statistical tests were performed. The stimulus features used in the present experiment matched the stimuli used in Experiment 3. Since patients showed a very good performance in identifying the stimulus shapes regardless of their size, we could exclude that the data obtained in Experiment 3 are driven by a poor perception of the large and small squares used as cues. Overall data indicate that the lack of a cue effect in patients reported in Experiment 3 cannot be accounted for by an inability to perceive the cue. 


\section{Discussion}

In the present paper, we explored the underlying mechanisms of simultanagnosia in posterior cortical atrophy with two main hypotheses: 1 ) the global processing deficit is due to an impairment in low-spatial frequency processing; and 2) simultanagnosia is, at least in part, due to an impairment in adjusting the focus of attention. This selective attention mechanism allows adjustment of the spatial extension of the attentional focus to either segregate small elements or include many elements in a global shape.

We tested five PCA patients, four of whom showed severe simultanagnosia being unable to report the global element of hierarchical letters, despite an intact ability to perceive local letters (Experiment 1) or same-size solid letters (Experiment 2). A perceptual manipulation was used in Experiment 1 to test the hypothesis according to which simultanagnosia is the consequence of a deficit in processing low-spatial frequencies (Thomas et al., 2012). There was mixed evidence of the effect of parvocellular-biased stimuli; performance was improved in one patient, worsened in another, and there was no evidence that performance improved or worsened in the others.

Experiment 3 investigated the impairment of focal attention in a detection task. Results suggest that while age- and education-matched controls were faster in responding to stimuli preceded by a red dot and small squared cues, four out of five PCA patients did not show any beneficial effect from the small-size cue. Remarkably, we found that healthy older participants did not show the same effects described by Albonico et al $(2016 ; 2017)$ in younger individuals. Results suggest that ageing affects the timing required for the activation of the fast, involuntary exogenous mechanism. Indeed, while short timing (e.g., $100 \mathrm{~ms}$ SOA) is sufficient to observe the effect of focal attention in younger participants (Albonico et al., 2016), longer timing are necessary for elderly healthy controls. We have also showed that such mechanism is impaired in PCA individuals, who did not show the focal 
attention effect at all. Furthermore, results of Experiment 3 showed that PCA patients, differently from controls, did not benefit from the red dot cue. Finally, experiment 4 showed that patients were highly accurate in identifying shapes of different size, suggesting that the lack of a cue effect in Experiment 3 is not the consequence of a poor perception of the cue itself.

Within the literature on simultanagnosia, the fracture between studies looking at the perceptual component and those exploring the attentional counterpart is significant. The present paper grounds on the conceptualization of simultanagnosia as the result of a complex interaction between attentional mechanisms and the perceptual processing, both partially or profoundly compromised in individuals with posterior cortical damage, such as PCA.

The hypothesis suggested by Thomas et al. (2012) is only in part supported by the present results, but they indicate that simultanagnosia is caused by, at least, two different mechanisms in PCA. Here we propose that, over and above the perceptual deficit, the second impaired mechanism has to do with an attentional component. While the absence of a benefit for the orienting cue (red dot) is representative of the spatial disorientation that is very common in this population (Lehmann et al., 2011), the lack of a small cue benefit requires a different interpretation, namely focal attention. This is a very new result in the literature on cognitive deficits in posterior cortical atrophy. Focal attention is normally modulated by exogenous factors, such as the dimension and features of the stimulus to be processed or the specific task requirements (Castiello \& Umiltà, 1990; Albonico et al., 2016; Albonico et al., 2017). Such features allow a flexible adjustment of the focus of attention-to local features or, instead, to global elements. Global processing is profoundly impaired in PCA as shown by deficits in object recognition (Kinsbourne \& Warrington, 1962), identification of hierarchical letters (Huberle \& Karnath, 2006) and complex scene exploration (Shakespeare et al., 2013). In each of these tasks, the focus of attention needs to be adjusted according to the stimulus' features and 
the specific task requirements. Here we suggest that a focal attentional deficit might interact with the core visuo-perceptual and visuo-spatial deficits typical of PCA in causing the phenomenology of simultanagnosia. Indeed, the only patient who did not have simultanagnosia in Experiment 1 (Patient 5) did not show any cue benefit in the focal attention test. However, such a patient was relatively less impaired in the visuo-perceptual tests (Table 1) as compared to the other patients. This result seems to indicate that a deficit in focal attention might be mitigated by relatively preserved perceptual abilities, further adding on the hypothesis of a mutual interaction between perceptual and attentional mechanisms in PCA.

The attentional interpretation of simultanagnosia as a "restricted spotlight of attention" (Dalrymple et al., 2010; Dalrymple et al., 2011) is somehow difficult to be reconciled with three main sources of evidence coming from both the literature and the present study: 1) improved performance with dense local element in the context of a same-size global stimuli (Huberle \& Karnath, 2006; Montoro et al., 2011); 2) lack of benefit from the small-size cue (Experiment 3 of the present study) and 3) accurate performance with non-complex stimuli vs. impaired performance with same-size complex stimuli (Experiments 1 and 2). Our interpretation of the attentional deficit linked to simultanagnosia in posterior cortical atrophy is that the focus of attention rather than being small in size is impaired in terms of its flexibility hindering both the focus shrinkage (lack of benefit from the small-size cue) and the focus enlargement (necessary for the adequate processing of complex stimuli). Such an interpretation also fits well with the description of the mechanisms involved in simultanagnosia by Riddoch and Humphreys (2004), who showed that patients found it easier to identify drawings of stimuli that could be recognised from their global shape than stimuli that required to rely on their internal details for recognition. 
In terms of anatomical correlates, several studies have revealed that the lateral and medial inferior parietal cortex is involved in global processing which is impaired in simultanagnosia (Coslett \& Saffran, 1991; Karnath, Ferber, Rorden, \& Driver, 2000; Rizzo \& Vecera, 2002; Nestor, Caine, Fryer, Clarke, \& Hodges, 2003; Himmelbach, Erb, Klockgether, Moskau, \& Karnath, 2009). In an fMRI study, Chen, Marshall, Weidner, and Fink, (2009a) have shown that, despite some differential activation, a common right posterior temporal-occipital-parietal network is involved in the update of the spatial information that allows the alternation between "zooming in and zooming out". The same brain network is normally reported as involved in spatial attention (Chen et al., 2009a; Chelazzi \& Corbetta, 2000) and is, at least in part, severely damaged in posterior cortical atrophy (Mendez, Ghajarania, \& Perryman, 2002; Nestor et al., 2003; Lehmann et al., 2011; Lehmann et al., 2012; (Yong et al., 2014). Such results in the literature suggests that particular brain networks, frequently damaged in PCA individuals, are strictly linked to the phenomenology of simultanagnosia. The small sample size of patients involved in the present study and the specific nature of their neurodegenerative condition limits broad generalization of the results and speculations about the brain circuits involved in simultanagnosia. Indeed, we cannot exclude that the visuo-perceptual deficit, particularly prominent in PCA rather than in other neuropsychological conditions, might interact with and modulate the features of simultanagnosia. Future studies will need to involve patients with simultanagnosia due to both PCA and other neurological conditions (e.g., stroke).

From a clinical perspective, simultanagnosia likely exacerbates text-reading difficulties (Yong et al., 2015), where patients report getting lost on a page of text, and so understanding to what extent perceptual or attentional mechanisms contribute to simultanagnosia is important in developing tailored interventions. 
In conclusion, in the present paper, we explored the hypothesis that simultanagnosia in posterior cortical atrophy grounds on two different but strictly linked mechanisms: a perceptual and an attentional one. We partially replicated the finding according to which the global processing deficit is related to the impairment in low-spatial-frequency processing and, more importantly, for the first time, we reported a focal attention deficit in posterior cortical atrophy. We claim that the inability to adjust the focus of attention according to the stimulus size might contribute to the impairment of global processing in PCA. Further research is needed in order to clarify the relative contribution of the perceptual and attentional mechanisms. 


\section{Figure caption:}

Figure 1. Examples of stimuli used for Experiment 1. Top row: unfragmented stimuli; bottom row: fragmented stimuli. Left: unbiased; right: parvocellular biased.

Figure 2. Example of a trial procedure in Experiment 3. After a blank screen (1000 ms), the target appearance could be preceded by no cue, red dot, small cue or big cue (in the interest of readability, in the figure only the last two conditions are shown). After 100 or $500 \mathrm{~ms}$, the target (T) was presented, and the participant was asked to respond as quickly as possible by pressing the space bar. 


\section{Bibliography}

Albonico, A., Malaspina, M., Bricolo, E., Martelli, M., \& Daini, R. (2016). Temporal dissociation between the focal and orientation components of spatial attention in central and peripheral vision. Acta Psychologica, 171, 85-92. https://doi.org/10.1016/j.actpsy.2016.10.003

Albonico, A., Malaspina, M., \& Daini, R. (2017). Target Type Modulates the Effect of Task Demand on Reflexive Focal Attention. Vision, 1(2), 13. https://doi.org/10.3390/vision1020013

Albonico, A., Martelli, M., Bricolo, E., Frasson, E., \& Daini, R. (2018). Focusing and orienting spatial attention differently modulate crowding in central and peripheral vision. Journal of vision, 18(3), 4-4.

Aresi, A., \& Giovagnoli, A. R. (2009). The role of neuropsychology in distinguishing the posterior cortical atrophy syndrome and Alzheimer's disease. Journal of Alzheimer's Disease, 18(1), 65-70.

Baxter, D. M., \& Warrington, E. K. (1985). Category specific phonological dysgraphia. Neuropsychologia, 23(5), 653-666.

Benson D, Davis R, \& Snyder BD. (1988). POsterior cortical atrophy. Archives of Neurology, 45(7), 789-793. https://doi.org/10.1001/archneur.1988.00520310107024

Castiello, U., \& Umiltà, C. (1990). Size of the attentional focus and efficiency of processing. Acta psychologica, 73(3), 195-209.

Charles, R. F., \& Hillis, A. E. (2005). Posterior cortical atrophy: Clinical presentation and cognitive deficits compared to Alzheimer's disease. Behavioural neurology, 16(1), 15-23.

Chelazzi, L., \& Corbetta, M. (2000). Cortical mechanisms of visuospatial attention in the primate brain. The new cognitive neurosciences, 667-686.

Chen, Q., Marshall, J. C., Weidner, R., \& Fink, G. R. (2009). Zooming In and Zooming Out of the Attentional Focus: An fMRI Study. Cerebral Cortex, 19(4), 805-819. https://doi.org/10.1093/cercor/bhn128

Chun, M. M., Golomb, J. D., \& Turk-Browne, N. B. (2011). A taxonomy of external and internal attention. Annual review of psychology, 62, 73-101.

COSLETT, H. B., \& SAFFRAN, E. (1991). Simultanagnosia: To see but not two see. Brain, 114(4), 1523-1545. 
Crawford, J. R., Garthwaite, P. H., \& Porter, S. (2010). Point and interval estimates of effect sizes for the case-controls design in neuropsychology: Rationale, methods, implementations, and proposed reporting standards. Cognitive neuropsychology, 27(3), 245-260.

Crutch, S. J., Lehmann, M., Gorgoraptis, N., Kaski, D., Ryan, N., Husain, M., \& Warrington, E. K. (2011). Abnormal visual phenomena in posterior cortical atrophy. Neurocase, 17(2), 160-177.

Crutch, S. J., Lehmann, M., Warren, J. D., \& Rohrer, J. D. (2013). The language profile of posterior cortical atrophy. Journal of Neurology, Neurosurgery \& Psychiatry, 84(4), 460-466.

Crutch, S. J., Schott, J. M., Rabinovici, G. D., Murray, M., Snowden, J. S., van der Flier, W. M., Dickerson, B. C., Vandenberghe, R., Ahmed, S., Bak, T. H., \& others. (2017). Consensus classification of posterior cortical atrophy. Alzheimer's \& Dementia.

http://www.sciencedirect.com/science/article/pii/S1552526017300407

Dalrymple, K. A., Birmingham, E., Bischof, W. F., Barton, J. J. S., \& Kingstone, A. (2011). Experiencing simultanagnosia through windowed viewing of complex social scenes. Brain Research, 1367, 265277. https://doi.org/10.1016/j.brainres.2010.10.022

Dalrymple, K. A., Bischof, W. F., Cameron, D., Barton, J. J. S., \& Kingstone, A. (2010). Simulating simultanagnosia: Spatially constricted vision mimics local capture and the global processing deficit. Experimental Brain Research, 202(2), 445-455. https://doi.org/10.1007/s00221-009-2152-3

Dalrymple, K. A., Kingstone, A., \& Barton, J. J. S. (2007). Seeing trees OR seeing forests in simultanagnosia: Attentional capture can be local or global. Neuropsychologia, 45(4), 871-875. https://doi.org/10.1016/j.neuropsychologia.2006.07.013

Duncan, J., Bundesen, C., Olson, A., Humphreys, G., Ward, R., Kyllingsbæk, S., van Raamsdonk, M., Rorden, C., \& Chavda, S. (2003). Attentional Functions in Dorsal and Ventral Simultanagnosia. Cognitive Neuropsychology, 20(8), 675-701. https://doi.org/10.1080/02643290342000041

Efron, R. (1969). What is Perception? In R. S. Cohen \& M. W. Wartofsky (A c. Di), Proceedings of the Boston Colloquium for the Philosophy of Science 1966/1968 (pagg. 137-173). Springer Netherlands. https://doi.org/10.1007/978-94-010-3378-7_4 
Epstein, J. N., Conners, C. K., Erhardt, D., March, J. S., \& Swanson, J. M. (1997). Asymmetrical hemispheric control of visual-spatial attention in adults with attention deficit hyperactivity disorder. Neuropsychology, 11(4), 467.

Eriksen, C. W., \& James, J. D. S. (1986). Visual attention within and around the field of focal attention: A zoom lens model. Perception \& psychophysics, 40(4), 225-240.

Eriksen, C. W., \& Yeh, Y. (1985). Allocation of attention in the visual field. Journal of Experimental Psychology: Human perception and performance, 11(5), 583.

Firth, N. C., Primativo, S., Marinescu, R.-V., Shakespeare, T. J., Suarez-Gonzalez, A., Lehmann, M., Carton, A., Ocal, D., Pavisic, I., Paterson, R. W., Slattery, C. F., Foulkes, A. J. M., Ridha, B. H., Gil-Néciga, E., Oxtoby, N. P., Young, A. L., Modat, M., Cardoso, M. J., Ourselin, S., ... Crutch, S. J. (2019). Longitudinal neuroanatomical and cognitive progression of posterior cortical atrophy. Brain. https://doi.org/10.1093/brain/awz136

Folstein, M. F., Folstein, S. E., \& McHugh, P. R. (1975). «Mini-mental state». A practical method for grading the cognitive state of patients for the clinician. Journal of Psychiatric Research, 12(3), 189-198.

Himmelbach, M., Erb, M., Klockgether, T., Moskau, S., \& Karnath, H.-O. (2009). FMRI of global visual perception in simultanagnosia. Neuropsychologia, 47(4), 1173-1177. https://doi.org/10.1016/j.neuropsychologia.2008.10.025

Huberle, E., \& Karnath, H.-O. (2006). Global shape recognition is modulated by the spatial distance of local elements-Evidence from simultanagnosia. Neuropsychologia, 44(6), 905-911. https://doi.org/10.1016/j.neuropsychologia.2005.08.013

Hughes, H. C., Fendrich, R., \& Reuter-Lorenz, P. A. (1990). Global Versus Local Processing in the Absence of Low Spatial Frequencies. Journal of Cognitive Neuroscience, 2(3), 272-282. https://doi.org/10.1162/jocn.1990.2.3.272

Jackson, M., \& Warrington, E. K. (1986). Arithmetic skills in patients with unilateral cerebral lesions. Cortex, 22(4), 611-620. 
James, M., Plant, G. T., \& Warrington, E. K. (2001). Cortical Vision Screening Test (Corvist). Suffolk, UK: Thames Valley Test Company.

Karnath, H.-O., Ferber, S., Rorden, C., \& Driver, J. (2000). The fate of global information in dorsal simultanagnosia. Neurocase, 6(4), 295-306.

Kinsbourne, M., \& Warrington, E. K. (1962). A disorder of simultaneous form perception. Brain, 85(3), 461486.

Lehmann, M., Barnes, J., Ridgway, G. R., Ryan, N. S., Warrington, E. K., Crutch, S. J., \& Fox, N. C. (2012). Global gray matter changes in posterior cortical atrophy: A serial imaging study. Alzheimer's \& Dementia, 8(6), 502-512. https://doi.org/10.1016/j.jalz.2011.09.225

Lehmann, M., Barnes, J., Ridgway, G. R., Wattam-Bell, J., Warrington, E. K., Fox, N. C., \& Crutch, S. J. (2011). Basic visual function and cortical thickness patterns in posterior cortical atrophy. Cerebral cortex, bhq287.

Maringelli, F., \& Umiltà, C. (1998). The control of the attentional focus. European Journal of Cognitive Psychology.

Mazza, V. (2017). Simultanagnosia and object individuation. Cognitive neuropsychology, 34(7-8), 430-439.

McKenna, P., \& Warrington, E. K. (1980). Testing for nominal dysphasia. Journal of Neurology, Neurosurgery \& Psychiatry, 43(9), 781-788. https://doi.org/10.1136/jnnp.43.9.781

McMonagle, P., Deering, F., Berliner, Y., \& Kertesz, A. (2006). The cognitive profile of posterior cortical atrophy. Neurology, 66(3), 331-338.

Mendez, M. F., Ghajarania, M., \& Perryman, K. M. (2002). Posterior cortical atrophy: Clinical characteristics and differences compared to Alzheimer's disease. Dementia and Geriatric Cognitive Disorders, 14(1), 33-40. https://doi.org/58331

Montoro, P. R., Luna, D., \& Humphreys, G. W. (2011). Density, connectedness and attentional capture in hierarchical patterns: Evidence from simultanagnosia. Cortex, 47(6), 706-714. https://doi.org/10.1016/j.cortex.2010.05.007 
Navon, DAVID. (1969). Forest before trees: The precedence of global features in visual perception.

Perception and Psychophysics, 5, 197-200.

Navon, David. (2003). What does a compound letter tell the psychologist's mind? Acta psychologica, 114(3), 273-309.

Nestor, P. J., Caine, D., Fryer, T. D., Clarke, J., \& Hodges, J. R. (2003). The topography of metabolic deficits in posterior cortical atrophy (the visual variant of Alzheimer's disease) with FDG-PET. Journal of Neurology, Neurosurgery \& Psychiatry, 74(11), 1521-1529.

Orpwood, E. K. W. P. M. L. (1998). Single Word Comprehension: A Concrete and Abstract Word Synonym Test. Neuropsychological Rehabilitation, 8(2), 143-154. https://doi.org/10.1080/713755564

Pavese, A., Coslett, H. B., Saffran, E. M., \& Buxbaum, L. J. (2002). Limitations of attentional orienting: Effects of abrupt visual onsets and offsets on naming two objects in a patient with simultanagnosia. Neuropsychologia, 40, 1097-1103. https://doi.org/10.1016/S0028-3932(01)00139-7

Renner, J. A., Burns, J. M., Hou, C. E., McKeel, D. W., Storandt, M., \& Morris, J. C. (2004). Progressive posterior cortical dysfunction A clinicopathologic series. Neurology, 63(7), 1175-1180.

Riddoch, M. J., \& Humphreys, G. W. (2004). Object identification in simultanagnosia: When wholes are not the sum of their parts. Cognitive Neuropsychology, 21(2-4), 423-441. https://doi.org/10.1080/02643290342000564

Rizzo, M., \& Vecera, S. P. (2002). Psychoanatomical substrates of Balint's syndrome. J Neurol Neurosurg Psychiatry, 72(2), 162-178.

Robertson, L. C. (1996). Attentional persistence for features of hierarchical patterns. Journal of Experimental Psychology: General, 125(3), 227.

Russell, C., Malhotra, P., \& Husain, M. (2004). Attention modulates the visual field in healthy observers and parietal patients. Neuroreport, 15(14), 2189-2193.

Shakespeare, T. J., Yong, K. X. X., Frost, C., Kim, L. G., Warrington, E. K., \& Crutch, S. J. (2013). Scene perception in posterior cortical atrophy: Categorization, description and fixation patterns. Frontiers in Human Neuroscience, 7. https://doi.org/10.3389/fnhum.2013.00621 
Stark, M. (1997). A restricted «spotlight» of attention in visual object recognition. Neuropsychologia, 35(9), 1233-1249. https://doi.org/10.1016/S0028-3932(97)00049-3

Tang-Wai, D. F., Graff-Radford, N. R., Boeve, B. F., Dickson, D. W., Parisi, J. E., Crook, R., Caselli, R. J., Knopman, D. S., \& Petersen, R. C. (2004). Clinical, genetic, and neuropathologic characteristics of posterior cortical atrophy. Neurology, 63(7), 1168-1174.

Thomas, C., Kveraga, K., Huberle, E., Karnath, H.-O., \& Bar, M. (2012). Enabling global processing in simultanagnosia by psychophysical biasing of visual pathways. Brain, 135(5), 1578-1585. https://doi.org/10.1093/brain/aws066

Turatto, M., Benso, F., Facoetti, A., Galfano, G., Mascetti, G. G., \& Umiltà, C. (2000). Automatic and voluntary focusing of attention. Perception \& Psychophysics, 62(5), 935-952.

Warrington, E. K. (1996). The Camden Memory Tests. Psychology Press.

Warrington, E. K., \& James, M. (1988). Visual apperceptive agnosia: A clinico-anatomical study of three cases. Cortex, 24(1), 13-32.

Warrington, E. K., \& James, M. (1991). The visual object and space perception battery: VOSP. Pearson London.

Willison, J. R., \& Warrington, E. K. (1992). Cognitive retardation in a patient with preservation of psychomotor speed. Behavioural neurology, 5(2), 113-116.

Yong, K. X. X., Rajdev, K., Shakespeare, T. J., Leff, A. P., \& Crutch, S. J. (2015). Facilitating text reading in posterior cortical atrophy. Neurology, 85(4), 339-348. https://doi.org/10.1212/WNL.0000000000001782

Yong, K. X. X., Shakespeare, T. J., Cash, D., Henley, S. M. D., Warren, J. D., \& Crutch, S. J. (2014). (Con)textspecific effects of visual dysfunction on reading in posterior cortical atrophy. Cortex, 57, 92-106. https://doi.org/10.1016/j.cortex.2014.03.010 